\title{
Entorhinal-Hippocampal Connections and Object Memory in the Rat: Acquisition versus Retention
}

\author{
Norbert Vnek, ${ }^{1}$ Theresa C. Gleason, ${ }^{1}$ Lawrence F. Kromer, ${ }^{2}$ and Lawrence A. Rothblat ${ }^{1}$ \\ 'Department of Psychology, George Washington University, Washington, DC 20052 and ${ }^{2}$ Department of Cell \\ Biology, Georgetown University, Washington, DC 20007
}

Investigations of the neurobiology of memory using experimental animals have modeled many of the characteristic features of amnesia seen in human clinical populations. To examine long-term memory, however, animal models of amnesia often employ extended measures of acquisition, which stand in contrast to the retention measures used with humans. To determine the role of entorhinal-hippocampal circuitry on both information acquisition and longterm retention, rats with bilateral transections of the angular bundle were trained on three object discrimination problems and then retrained two weeks later to measure retention. Animals with discrete lesions of the angular bundle, which disrupted perforant path connections from the entorhinal cortex to the hippocampus and efferent hippocampal-cortical projections, acquired the object discrimination problems normally but showed a marked deficit in retention. These findings are important because they indicate that the role of entorhinal-hippocampal connections may be limited to maintaining some types of information (e.g., single object discriminations) for retention. This dissociation, moreover, suggests that behavioral paradigms that include a measure of retention may be particularly important for characterizing the mnemonic functions of the hippocampal/parahippocampal region.

[Key words: hippocampal formation, entorhinal cortex, parahippocampal region, memory, object discriminations, rats]

Much of what is presently known about temporal lobe involvement in memory has been borne by the study of patient populations who developed amnesia from a variety of etiologies. For example, patients treated for epilepsy by surgical removal of the hippocampus, amygdala, and adjacent cortices display severe and long lasting impairments of specific types of memories (Scoville and Milner, 1957; Corkin, 1984). The memory deficits seen in Alzhcimer's discase, morcover, have been attributed to ncuropathology in entorhinal cortex and hippocampus (Van Hoesen et al., 1991; Braak and Braak, 1993). However, because of the limitations inherent to the study of clinical populations, animal models of human amnesic syndromes have been developed to more precisely identify the neuroanatomical sites that contribute to memory functions.

Received June 9, 1994; revised Sept. 19, 1994; accepted Oct. 18, 1994

This work was supported by Office of Naval Research Grant N00014-88-K0227 and George Washington University Facilitating Fund.

Correspondence should be addressed to Dr. L. A. Rothblat at the above address.

Copyright (C) 1995 Society for Neuroscience $0270-6474 / 95 / 153193-07 \$ 05.00 / 0$
Studies using experimental animals have succeeded in modeling many - but not all_of the characteristic features of human amnesia. The success is largely due to the development of behavioral tasks that measure variables in animals that are analogous to those commonly examined in human clinical populations. For example, monkeys with medial temporal damage have consistently been found to be impaired on delayed-nonmatchingto-sample at longer delays (Mishkin, 1978; Mahut et al., 1982; Zola-Morgan and Squire, 1985, 1986), a working memory task, and on concurrent object discrimination (COD) (Mahut et al., 1982; Zola-Morgan and Squire, 1985), a long-term memory task. These object discrimination tasks, moreover, have been successfully adapted for use with rats and found to be sensitive to hippocampal or parahippocampal damage in this species as wcll (Rothblat ct al., 1993; Mumby and Pinel, 1994).

Object discrimination tasks have proven to be similar to memory tests used with humans, yet animal models using these and other paradigms have examined only a small subset of the mnemonic variables explored in human research. Specifically, in work with humans, subjects are required to memorize a list of items and then are tested on the retention of this list over delay intervals ranging from minutes (e.g., Graf and Mandler, 1984) to months (e.g., Tulving et al., 1991). In contrast, studies of longterm memory using experimental animals typically do not employ discrete acquisition and retention phases in training subjects on lists of items. For example on the COD task used to study long-term memory, animals are trained to criterion performance on multiple discriminations, a measure of acquisition, but generally are not tested on the degree to which this information is thereafter retained.

Thus, the present study was designed to examine the effects of disrupting entorhinal-hippocampal circuitry on both the acquisition and long-term retention of multiple object discriminations. We trained rats with bilateral angular bundle transections and controls on three successive object discrimination problems and then retrained them on the same problems 2 weeks later to measure retention. Angular bundle transections were chosen because they afford the opportunity to study the effects of entorhinal-hippocampal disconnection on memory in the absence of extensive damage to parahippocampal structures that also are important to memory (Bunsey and Eichenbaum, 1993; Meunier et al., 1993; Zola-Morgan and Squire, 1993).

\section{Materials and Methods}

Subjects. Nineteen male Wistar rats (Charles River, Wilmington, MA) served as subjects. All animals were approximately $90 \mathrm{~d}$ old and weighed between 300 and $400 \mathrm{gm}$ at the start of the study. Animals 
were housed individually in wire cages and deprived to $85 \%$ of body weight throughout behavioral testing.

Surgery. Eight rats received bilateral knife-cuts of the angular bundle, three rats received control knife-cut lesions of occipital neocortex superjacent to the angular bundle, and eight animals served as normal controls. Animals were deeply anesthetized with a mixture of ketamine (90 $\mathrm{mg} / \mathrm{kg}$ ) and xylazine (15 $\mathrm{mg} / \mathrm{kg}$, i.p.) during surgery, and lesion production proceeded under a surgical microscope.

Angular bundle $(\mathrm{AB})$ lesions were intended to transect the entire medial-lateral extent of the angular bundle, including both medial and lateral perforant path fibers. A surgical knife (Mini-Blade 6200; R. Beaver, Waltham, MA) was lowered into the brain bilaterally, with the skull in a level position, at the following stereotaxic coordinates: 7.8 $\mathrm{mm}$ posterior to bregma, $3.5 \mathrm{~mm}$ lateral to the midline, and $5.5 \mathrm{~mm}$ ventral to dura. The cortical control lesions (CC) were prepared by cutting the occipital neocortex that overlays the angular bundle; the coordinates for the $\mathrm{CC}$ lesion were identical to that of the $\mathrm{AB}$ lesion except the knife was lowered approximately $1 \mathrm{~mm}$ ventral to dura. Normal control (NC) animals did not undergo any surgical procedures.

Apparatus. Behavioral testing utilized an elevated $(65 \mathrm{~cm}$ in height) Y-maze, as previously described (Rothblat et al., 1993). The maze comprised a $90 \mathrm{~cm}$ long $\times 9 \mathrm{~cm}$ wide stem that divided at a $30^{\circ}$ angle into two $25 \mathrm{~cm} \times 7 \mathrm{~cm}$ goal arms. A $2 \mathrm{~cm}$ high lip was attached along the edges of the stem and goal arms to prevent animals from falling off the maze. A $7 \mathrm{~cm} \times 1 \mathrm{~cm} \times 1 \mathrm{~cm}$ ridge separated each goal arm from the stem, and $10 \mathrm{~cm}$ behind each ridge was a flat $(10 \mathrm{~cm} \times 7 \mathrm{~cm} \times 0.5$ $\mathrm{cnl}$ ) wooden insert with a $2 \mathrm{~cm}$ hole that served as a lood well. The center of the food well was located $13 \mathrm{~cm}$ behind the ridge, and immediately in front of the well were five small holes, each containing a $45 \mathrm{mg}$ food pellet (P. J. Noyes, Lancaster, $\mathrm{NH}$ ) that was inaccessible to the animal. These pellets were used to prevent the use of olfactory cues in learning the object discriminations. Animals were placed behind a removable $40 \mathrm{~cm} \times 20 \mathrm{~cm}$ free standing door $25 \mathrm{~cm}$ from the beginning of the stem between trials. The discrimination stimuli consisted of three pairs of "junk" objects, each mounted on a $3 \mathrm{~cm} \times 3 \mathrm{~cm}$ wood base. One object of each pair served as the correct stimulus for all animals.

Procedure. Animals who underwent surgery began training on three successive object discrimination problems after a 2 week recovery period. On a given trial, each junk object of a pair was placed over one of the food wells and the animal was placed behind the free standing door on the stem of the Y-maze. The door was then removed and the animal was allowed to traverse the stem and choose one of the stimuli. Two $(45 \mathrm{mg}$ ) food pellets were placed only under the correct object, and were accessible to the rat after the correct object was displaced. The objects of each pair were pseudorandomly assigned to the left and right goal arms across trials to preclude the use of spatial cues in learning the discriminations. An incorrect choice was determined to have been made when both of the animal's front paws crossed the ridge separating the stem from the goal arm holding the incorrect stimulus. After each trial, the animal was placed at the beginning of the stem, the free standing door was replaced, and a $10 \mathrm{sec}$ interval was imposed before the beginning of the next trial. All animals received 20 successful trials of training each day. To ensure successful completion of each trial a correction procedure was used when an incorrect choice was made (i.e., the trial was repeated until the correct object was chosen). Criterion was set to $90 \%$ correct responses (i.e., at least 18 correct first-response trials) on a given training session, and the dependent measure was the number of days of training necessary to reach criterion on a given problem. Training on the second and third problems began the day after reaching criterion on the preceding problem. Three different orders of the three pairs of objects were used and counterbalanced across groups.

A 2 week period intervened between the day the animal reached criterion on the third discrimination problem and retraining on the three discriminations. The problem order for retraining was the same as that of training for all animals, as was the general testing procedure.

Histology. Upon completion of retraining, animals were sacrificed and prepared for histochemical analyses. Animals were deeply anesthetized via intraperitoneal administration of nembutal and transcardially perfused with $4 \%$ paraformaldehyde in $0.1 \mathrm{~m}$ phosphate buffer $(\mathrm{pH} 7.4)$. Following removal, brains were postfixed in $4 \%$ buffered paraformaldehyde for $2 \mathrm{hr}$ and then equilihrated in $20 \%$ buffered sucrose. All brains were then sectioned horizontally with a cryostat, and two sets of $20 \mu \mathrm{m}$ sections in a one and eight series were retained for histology. One set of sections was stained with cresyl violet for histological verification of the lesions. The second set was reacted with a histochemical procedure (Tsuji, 1974) that stained for acetylcholinesterase (AChE) to exanine reorganization of hippocanpal cholinergic terninals following lesions, as well as to confirm deafferentation in animals with angular bundle lesions. Histological verification was achieved through comparison of cresyl violet stained sections with the normative representations in Paxinos and Watson's (1986) stereotaxic atlas.

\section{Results}

\section{Histological}

Histological analysis of cresyl violet stained material from cortical control animals revealed that these knife lesions were symmetrical and that they were confined to the posterior portion of area 18a. Moreover, the hippocampal formation and parahippocampal area were not damaged by these control lesions. Cresyl violet stained sections taken from animals with angular bundle transections indicated that the lesions were largely symmetrical and appropriately positioned. The lesions resulted in bilateral transections of the angular bundle that spared the dentate gyrus, Ammon's horn, and the subiculum in all animals (see Fig. 1A). Most specimens were found to have complete transections of the angular bundle at dorsal levels, although there was occasional sparing of the most lateral portion of the angular bundle. The knife cut included additional hilateral damage to lateral occipital cortex area 2 at dorsal levels, with variable unilateral damage to perirhinal and retrosplenial cortices. At more ventral levels, seven specimens demonstrated similar bilateral transections of the angular bundle, with one showing only a unilateral transection. Two specimens demonstrated bilateral damage to temporal cortex area 2, and one had bilateral lesions in perirhinal cortex. Variable unilateral damage to perirhinal cortex, presubiculum, and parasubiculum was also evident in some material. A schematic reconstruction of the angular bundle lesion in an animal displaying behavioral deficits typical of this group appears in Figure 2.

Angular bundle animals were also found to have an increased density of AChE staining in the outer two-thirds of the molecular layer of the dentate gyrus (see Fig. $1 B$ ). The increase in AChE staining represents the sprouting of AChE-positive septohippocampal fibers in regions of the molecular layer that were freed of perforant path synaptic contacts after deafferentation (Lynch et al., 1972; Cotman et al., 1973). Most specimens showed an increased density of AChE staining in both the superficial and middle thirds of the dentate molecular layer throughout the dorsoventral extent of the hippocampus, indicative of a loss of both medial and lateral perforant path axons. A few specimens, however, while demonstrating sprouting in the outer two-thirds of the dentate molecular layer dorsally, at ventral levels showed an expanded AChE-rich zone solely in the middle third, which represents a loss of only the medial perforant path (Stanfield and Cowan, 1982).

\section{Behavioral}

The performance of normal and cortical control groups was similar on both the training and retraining phases of testing. The mean number of days needed to reach criterion during training. ordered from the first discrimination learned (problem 1) to the last (problem 3), was $13.88(\mathrm{SEM}= \pm 1.68), 10.25( \pm 1.64)$, and $9.50( \pm 0.80)$ for $\mathrm{NC}$ animals, and $8.0( \pm 1.53), 9.67$ $( \pm 1.86)$, and $10.33( \pm 3.48)$ for the CC group. During retraining, NC scores on the three problems, again ordered chronologically, were $3.13( \pm 0.85), 1.88( \pm 0.23)$, and $1.88( \pm 0.40)$, compared to CC. scores of $2.33( \pm 0.88), 2.33( \pm 0.67)$, and 1.33 $( \pm 0.33)$. All of the following statistical analyses were first per- 

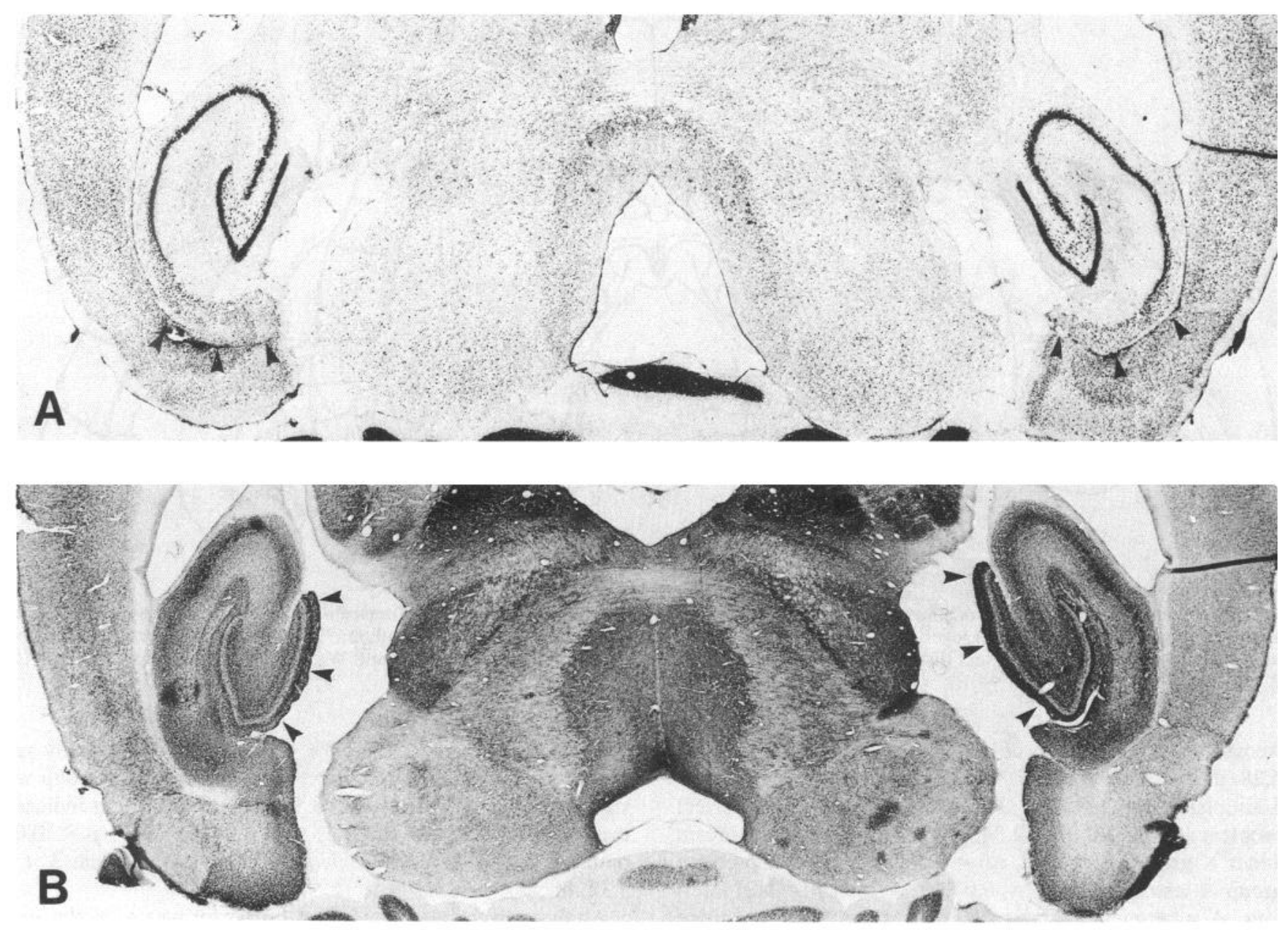

Figure 1. Photomicrographs of horizontal sections $(-4.74 \mathrm{~mm}$ from bregma) from specimen ANG8 illustrating the location of bilateral angular bundle lesions. Cresyl violet $(A)$ stained sections revealed symmetrical, bilateral transections of angular bundle axons, indicated by arrows. In this specimen, AChE $(B)$ stained material confirmed the loss of perforant path input from both medial and lateral entorhinal cortex as evinced by sprouting of AChE-positive fibers in both the middle and outer thirds of the dentate molecular layer (arrows).

formed on the behavioral data for normal control and cortical control animals, and confirmed that the two groups did not differ significantly on any of the behavioral measures $(p=\mathrm{NS})$. The scores for these animals were therefore combined for comparison with the scores of the lesioned animals. This pooled group will be referred to as the CON group.

The results of training on problems $1-3$ for $\mathrm{AB}$ and $\mathrm{CON}$ groups appear in Figure 3. Angular bundle animals required $14.25( \pm 2.58), 10.13( \pm 2.29)$, and $10.38( \pm 0.84)$ days to learn problems 1,2 , and 3 , respectively, whereas control animals achieved criterion in $12.27( \pm 1.50), 10.09( \pm 1.25)$, and 9.73 $( \pm 1.00) \mathrm{d}$ on problems $1-3$. However, retraining performance diverged, as control animals showed greater improvement than angular bundle animals. As illustrated in Figure 4, the mean number of days required to reach criterion during retraining on problems $1-3$ were $7.5( \pm 2.24), 5.0( \pm 1.82), 6.25( \pm 2.00)$ for the $\mathrm{AB}$ group and 2.91. $( \pm 0.65), 2.00( \pm 0.23)$, and 1.73 $( \pm 0.30)$ for the CON group.

A $2(\mathrm{AB}$ vs $\mathrm{CON}) \times 3$ (problem 1 vs problem 2 vs problem 3 ) $\times 2$ (testing phase; training vs retraining) repeated measures ANOVA showed a main effect of group $(F=8.19$, df $=1,17$, $p<0.05$ ), indicating that across problems and testing phases control animals learned the discriminations in fewer days than angular bundle animals. There was also a main effect of testing phase $(F=106.15$, df $=1,17, p<0.0001)$, where fewer days were required to reach criterion during retraining than were required for training, and a testing phase $\times$ group interaction $(F$ $=5.52$, df $=1,17, p<0.05)$. Analysis of the means revealed that this interaction was due to a difference between groups in performance at the retraining phase of testing but not at training, indicative of different levels of retention but not acquisition between groups. Angular bundle animals required a mean of 34.75 $\mathrm{d}( \pm 2.52)$ to complete testing on the three discrimination problems during training, which did not differ from the mean of 32.09 ( \pm 2.45$)$ needed by control animals. During the retraining phase, in contrast, the mean total number of days required by angular bundle animals to reach criterion on the three problems was $18.75( \pm 3.13)$, which differed from the mean of 6.64 $( \pm 1.05) \mathrm{d}$ achieved by controls. The main effect of testing phase indicated that, overall, there was an improvement in performance during the retraining phase of testing. This, coupled with the testing phase $\times$ group interaction, suggests that control animals performed significantly better at retraining than at training. To determine if this was also true for lesioned animals, a pairedsamples $t$ test was conducted on the difference between the training and retraining totals, across problems, and a significant im- 


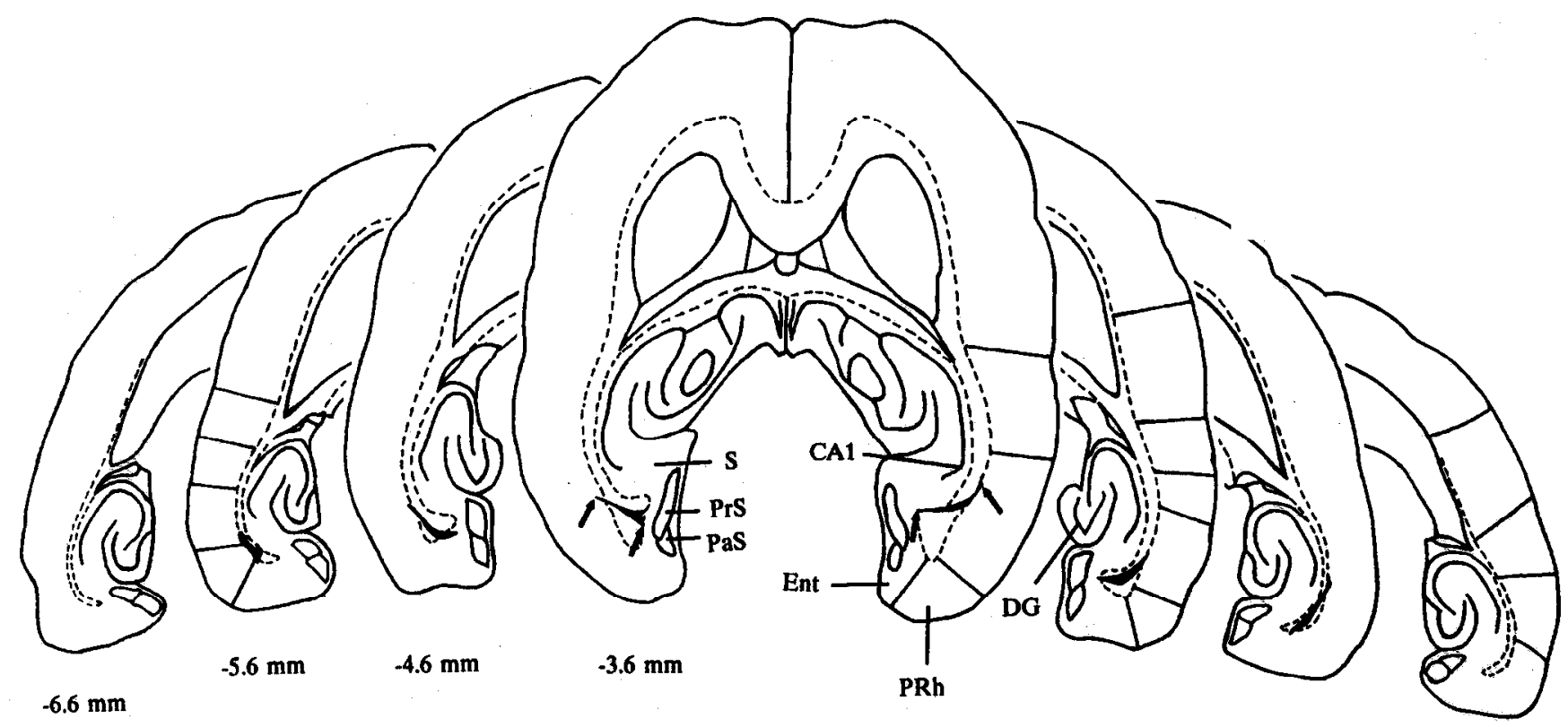

Figure 2. Schematic reconstruction of bilateral angular bundle transections from specimen ANG4. Transections are demarcated by arrows on the most dorsal ( $-3.6 \mathrm{~mm}$ from bregma) level. $D G$, dentate gyrus; $E n t$, entorhinal cortex; $P a S$, parasubiculum; $P R h$, perirhinal cortex; $P r S$, presubiculum; $S$, subiculum. Numbers represent distance from bregma. Adapted with permission from Paxinos and Watson, 1986 (their Figs. 99, 103, 107, 111).

provement in performance was evident for this group as well ( $t$ $=4.28$, df $=7, p<0.01$ ).

Additionally, the $2 \times 3 \times 2$ ANOVA showed a main effect of problem $(F=4.38$, df $=2,34, p<0.05)$, but not significant problem $\times$ group $(F=0.48$, df $=2,34, p=\mathrm{NS})$ or problem $\times$ group $\times$ testing phase $(F=0.04, \mathrm{df}=2,34, p=\mathrm{NS})$ interactions. A subsequent analysis (Sheffé test) revealed that more days were required to reach criterion on problem 1, across groups and testing phases, than were required for problems 2 or 3. It is likely that the first problem was the most difficult because subjects were becoming accustomed to the maze and procedure whilc lcarning it.

To ensure that a difference between $A B$ and $C O N$ groups during acquisition was not occluded by the correction procedure, a $2 \times 3$ repeated measures ANOVA was conducted on the number of trials needed to reach criterion on the three discrimination problems during the training phase of testing. This analysis confirmed that the two groups did not differ on acquisition, as the main effect of group was not significant $(F=1.66$, dr $=1,17$, $p=\mathrm{NS})$.

To further examine the difference between $\mathrm{AB}$ and $\mathrm{CON}$ groups on retention, performance on the first day of retraining on each of the discrimination problems was analyzed. On the first day of retraining, $\mathrm{AB}$ animals made 14.25 ( \pm 1.06 ), 14.75 $( \pm 1.05)$, and $14.50( \pm 0.85)$ correct choices on problems $1-3$, for an overall mean of $14.50( \pm 0.57)$ correct trials and an average accuracy of $72.50 \%$. In contrast, CON animals achieved $16.0( \pm 0.75), 16.45( \pm 0.62)$, and $16.64( \pm 0.80)$ correct trials on the first day of retraining on problems $1-3$, for an overall mean of $16.36( \pm 0.56)$ and $81.82 \%$ accuracy. A $2 \times 3$ repeated measures ANOVA on these data confirmed that, across problems, the CON group made significantly more correct choices on the first day of retraining than did the $\mathrm{AB}$ group, as a significant main effect of group was found $(F=5.16$, df $=1,17$, $p<0.05)$. Although the performance of control animals exceeded that of lesioned animals on the first day of retraining, the average accuracy of $72.50 \%$ achieved by $\mathrm{AB}$ animals suggested that the number of correct trials made by this group was significantly greater than chance. Paired-samples $t$ tests indicated that this was true (problem 1: $t=3.99$, df $=7, p<0.01$; problem 2: $t=4.53$, df $=7, p<0.01$; and problem $3: t=$ 5.32, df $=7, p<0.01$ ).

Analyses were also conducted on performance on the individual pairs of objects used for the three discrimination problems, independent of position in testing order, to determine if either group was differentially sensitive to any of the three pairs. Training and retraining scores for the three pairs of objects were subjected to a $2 \times 3 \times 2$ repeated mcasures ANOVA, which failed to find a main effect of object pair $(F=1,85$, df $=2,34$, $p=\mathrm{NS})$, or a significant object pair $\times$ group interaction $(F=$ 0.49 , df $=2,34, p=\mathrm{NS}$ ), suggesting that angular bundle and control animals were not differentially affected by the individual object pairs used for the three discrimination problems.

\section{Discussion}

Rats with transections of the angular bundle demonstrated normal acquisition but impaired retention of three visual discrimination problems. That is; although lesioned animals acquired the three discrimination problems in the same amount of time as did controls, they showed a markedly reduced benefit from prior learning during the retraining phase of testing. It has previously been shown that rats with hippocampal/parahippocampal damage are impaired on retention, but not acquisition, on simple brightness discrimination tasks (Myhrer and Iversen, 1990; Levisohn and Isacson, 1991). Additionally, rats with entorhinal cortex lesions have demonstrated an impaired ability to retain an olfactory discrimination that was normally acquired one or three hours earlier (Staubli et al., 1984, 1986). The present results extend this acquisition-retention distinction to performance on object discrimination tasks that have proven to be highly amnesia-sensitive in humans and monkeys (Squire et al., 1988). Taken together, these findings suggest that the long-term reten- 
$\square$ Angular Bundle $\square$ Control

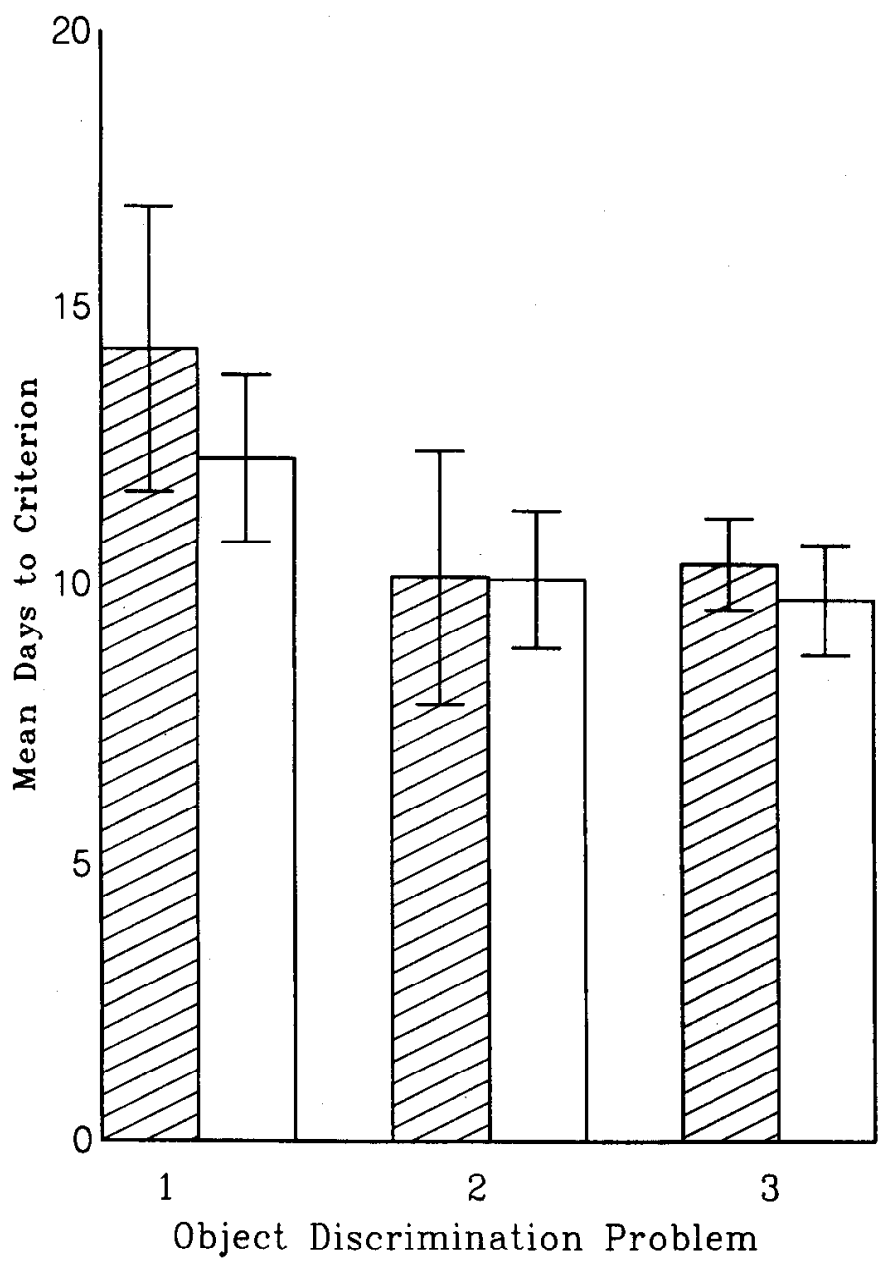

Figure 3. Performance of animals with angular bundle transections and controls on the training phase (acquisition) of testing on threc object discrimination problems. Problem 1 was the first learned discrimination; Problem 3, the last learned discrimination. Performance on acquisition did not differ between the two groups. Error bars, SEM.

tion of information is more critically dependent upon entorhinalhippocampal connections than information acquisition. The deficit in retaining object discriminations over a 2 week interval seen in angular bundle animals is also in accord with the impaired retention of single object discriminations found in monkeys with medial temporal lobe lesions (Zola-Morgan and Squire, 1984, 1985).

Another important finding from the present study is that discrete lesions of the angular bundle can effect a strong behavioral impairment. The hippocampal formation and adjacent cortices occupy a large region in the rat brain; thus, traditional aspiration lesions of these structures or their components produce, by necessity, rather extensive damage. Angular bundle transections, in contrast, are relatively small in size and cause minimal damage to surrounding structures. That such a restricted lesion can induce a deficit on an object discrimination task emphasizes the importance of entorhinal-hippocampal circuitry to memory.

The preserved capacity of angular bundle lesioned animals to acquire the object discriminations may have been due to the nature of the information measured by this task. Some object

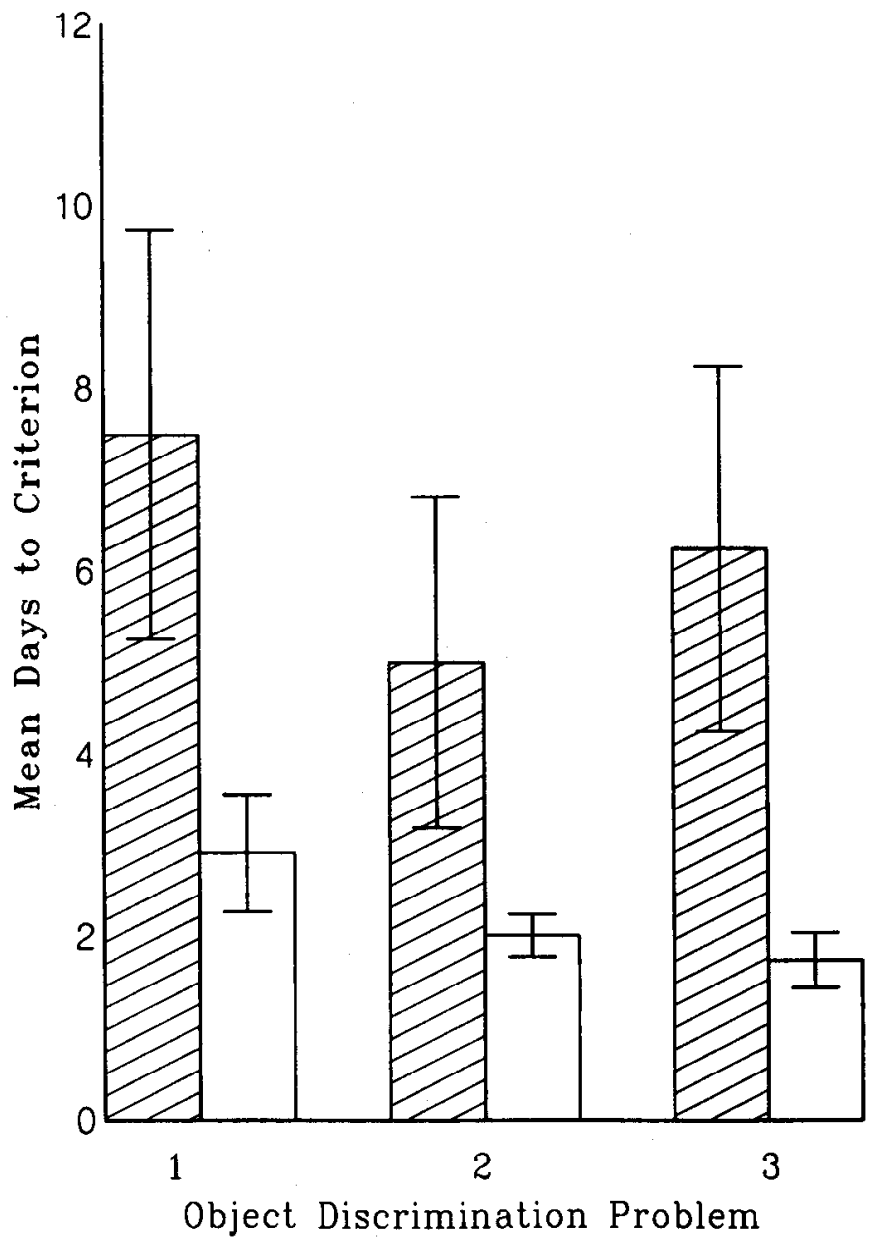

Figure 4. Performance of animals with angular bundle transections and controls on the retraining phase (retention) of testing on three object discrimination problems. Across problems, angular bundle animals required significantly more days to reach criterion than controls. Error bars, SEM.

discrimination tasks (e.g., $24 \mathrm{hr}$ concurrent discrimination learning; Malamut et al., 1984) have been found to be insensitive to hippocampal/parahippocampal lesions, presumably because they involve the formation of stimulus-response associations that are noncognitive habits rather than declarative memories. The acquisition of habits is thought to be the domain of a corticostriatal information processing circuit and thus invulnerable to hippocampal/parahippocampal injury. Behavioral tasks that tap the habit system involve repeated exposure to a limited set of stimuli, where each correct stimulus and the choice response are formed into an associative bond by food reinforcement (Mishkin et al., 1984). The object discrimination task used in the present study has these properties. Thus, the normal performance of lesioned animals during training may have been mediated by the corticostriatal habit system, which was intact in angular bundle animals.

In contrast to the spared ability of angular bundle transected animals to acquire information in the present study, we recently found that both excitotoxic (NMDA) and aspiration lesions of the entorhinal cortex grossly impair the acquisition of a concur- 
rent object discrimination task (COD) using four intermingled pairs of stimuli (Rothblat et al., 1993). Aside from using four rather than three pairs of objects and training rats to learn the discriminations concurrently, the protocols for the COD task and that of the present study were identical. The differences in the protocols, however, are significant because concurrent training elevates the degree of interitem interference and a greater stimulus set size increases the memory load of the task. Additionally, learning multiple discriminations concurrently is more difficult than single discrimination learning because it imposes a greater delay between presentations of discriminative stimuli. In single discrimination learning there is a negligible delay between stimuli repetitions, which makes the training experience considerably more intensive than that of COD learning. These parametershigh levels of interference, a delay between stimuli repetitions, and a large memory load-augment the task demands of the COD relative to single discrimination learning.

Behavioral paradigms with high task demands, particularly those. with substantial levels of interitem interference, are thought to necessitate the mnemonic processing functions (e.g., pattern separation and the formation of relational representations) of hippocampal/parahippocampal structures for successful acquisition (Shapiro and Olton, in press). Consistent with this view and the present findings, Wible et al. (1992) found that rats with lesions of the fornix-fimbria or hippocampus showed normal acquisition of a single object discrimination problem but impaired acquisition on an eight-pair COD task. Although the paradigm used in the present study did not require concurrent training, multiple discrimination problems were used, which even in the absence of intermingled organization likely led to proactive interference and thus relatively high task demands. It is therefore somewhat surprising that entorhinal-hippocampal disconnection did not affect acquisition performance. Thus, although some characteristics of the present visual discrimination paradigm are consistent with those considered to be habit tasks, the extent to which it can be so classified is unclear.

The impaired retention of the visual discrimination problems demonstrated by angular bundle animals suggests that entorhinal-hippocampal circuitry, while not involved in the acquisition of stimulus-response associations, may nonetheless play a role in the retention of such information. Therefore, although the corticolimbic and corticostriatal systems are involved in fundamentally different types of learning, these two systems may not be entirely independent. Specifically, the functional properties of the corticostriatal system are uniquely suited for establishing stimulus-response associations because this system allows for the interaction of sensory neocortex and motor basal ganglia (Mishkin et al., 1984). However, when simple stimulus-response associations are learned under conditions of high task demands, the maintenance and retention of this information may require the special information processing and neuroplastic capacities of the hippocampal formation (Squire, 1992; Shapiro and Olton, in press). Under such conditions, such as interference among a list of items, habits may need to be cycled through the hippocampal formation and undergo consolidation in order to be retained at optimal strength. Angular bundle transections, which damage entorhinal-hippocampal interconnections, must have compromised information consolidation in lesioned animals. Reduced consolidation capacities may have limited the strength of information storage and thus led to a retention deficit.

The dissociation between the acquisition and retention of object discriminations demonstrated by angular bundle animals stands in contrast to the more global deficits seen in rats with hippocampal damage on traditional spatial measures of memory. For example, rats with hippocampal lesions are impaired on both the acquisition and long-term retention of spatial information (Sara and David-Remacle, 1981; Peinado-Manzano, 1990). That hippocampal lesions lead to both acquisition and retention impairments on measures of spatial memory likely reflects the declarative nature of this information (Squire, 1992).

What is also compelling about the performance of lesioned animals in the present study is that despite demonstrating impaired retention, these animals still showed a significant degree of savings of the three discrimination problems. Across problems, animals with angular bundle transections required roughly half as many days to reach criterion on each problem during the retraining phase of testing as were required during training (mean days-to-criterion at training was 11.58 and at retraining was 6.25 ). The capacity of lesioned animals to retain visual discriminations over a 2 week interval may have been due to variability in the completeness of angular bundle lesions. The variable sparing of ventral perforant path axons may have been sufficient to allow for some maintenance or consolidation of information, although not of the efficacy possible in normal animals. Alternatively, the retention demonstrated by lesioned animals may have been mediated by the corticostriatal system. Specifically, it may be that the habit system is capable of maintaining information for retention, even when task demands are high, but can not do so as effectively as the corticolimbic declarative system.

The present findings demonstrate the importance of expanding the range of mnemonic variables that are examined in animal models of amnesia. Traditionally, animal research has focused on the effects of lesions on information acquisition. This emphasis on acquisition may limit the sensitivity of behavioral measures of memory and mask some lesion effects, as would have been the case in the present study had retention not been assessed. Measures of retention, moreover, avail themselves to manipulating delay intervals, a key variable in human memory research. Although the effects of delay intervals have been studied in short-term memory tasks (e.g., delayed-nonmatching-tosample), this variable has largely been ignored in lesion studies of long-term memory. Examining delay effects and other variables that are commonly manipulated in human research will help to better characterize the nature of lesion-induced amnesia in animals. Additionally, this strategy will facilitate comparisons between human amnesic syndromes and paradigms designed to model them in animals.

Our results indicate that circumscribed lesions of the angular bundle are sufficient to induce a profound amnesia for object discriminations in rats. The behavioral effects of such a discrete lesion, however, are manifest as a retention but not an acquisition deficit. This dissociation underscores the importance of examining a variety of mnemonic variables in animal research, a strategy that may expose latent behavioral deficits and aid in the development of more complete models of human amnesic syndromes.

\section{References}

Braak H, Braak E (1993) Entorhinal-hippocampal interaction in mnestic disorders. Hippocampus 3:239-246.

Bunsey M, Eichenbaum $\mathbf{H}$ (1993) Critical role of the parahippocampal region for paired-associate learning in rats. Behav Neurosci 107:740747.

Corkin S (1984) Lasting consequences of bilateral medial temporal 
lobectomy: clinical course and experimental findings in H.M. Semin Neurol 4:249-259.

Cotman CW, Matthews DA, Taylor D, Lynch G (1973) Synaptic rearrangement in the dentate gyrus: histochemical evidence of adjustments after lesions in immature and adult rats. Proc Natl Acad Sci USA 70:3473-3477.

Graf $P$, Mandler G (1984) Activation makes words more accessible, but not necessarily more retrievable. J Verb Learn Verb Behav 23: 553-568.

Levisohn LF, Isacson O (1991) Excitotoxic lesions of the rat entorhinal cortex. Effects of selective neuronal damage on acquisition and retention of a non-spatial reference memory task. Brain Res 564:230244.

Lynch G, Matthews DA, Mosko S, Parks T, Cotman C (1972) Induced acetylcholinesterase-rich layer in rat dentate gyrus following entorhinal lesions. Brain Res 42:311-318.

Mahut H, Zola-Morgan S, Moss M (1982) Hippocampal resections impair associative learning and recognition memory in the monkey. J Neurosci 2:1214-1229.

Malamut BL, Saunders RC, Mishkin M (1984) Monkeys with combined amygdalo-hippocampal lesions succeed in object discrimination learning despite 24-hour intertrial intervals. Behav Neurosci 98 : 759-769.

Meunier M, Bachevalier J, Mishkin M, Murray EA (1993) Effects on visual recognition of combined and separate ablations of the entorhinal and perirhinal cortex in rhesus monkeys. J Neurosci 13:54185432.

Mishkin M (1978) Menury in monkeys severely impaired by conbined but not by separate removal of amygdala and hippocampus. Nature 273:297-298.

Mishkin M, Malamut BL, Bachevalier J (1984) Memories and habits: two neural systems. In: Neurobiology of learning and memory (Lynch G, McGaugh JL, Weinberger NM, eds), pp 65-77. New York: Guilford.

Mumby DG, Pinel JPJ (1994) Rhinal cortex lesions and object recognition in rats. Behav Neurosci 108:11-18.

Myhrer T, Iversen EG (1990) Changes in retention of a visual discrimination task following unilateral and bilateral transections of temporoentorhinal connections in rats. Brain Res Bull 25:293-298.

Paxinos G, Watson C (1986) The rat brain in stereotaxic coordinates. San Diego: Academic.

Peinado-Manzano MA (1990) The role of the amygdala and the hippocampus in working memory for spatial and non-spatial information. Behav Brain Res 38:117-134.

Rothblat LA, Vnek N, Gleason TC, Kromer LF (1993) Role of the parahippocampal region in spatial and non-spatial memory: effects of parahippocampal lesions on rewarded alternation and concurrent object discrimination learning in the rat. Behav Brain Res 55:93-100.

Sara SJ, David-Remacle M (1981) Discriminative avoidance lcarning in hippocampal and cortical rats: acquisition rate, behavioral strategies, and long-term retention. Physiol Psychol 9:37-48.

Scoville WB, Milner B (1957) Loss of recent memory after bilateral hippocampal lesions. J Neurol Neurosurg Psychiatry 20:11-21.

Shapiro ML, Olton DS (in press) Hippocampal function and interference. In: Memory systems 1994 (Schacter DL, 'Tulving E, eds), in press. Cambridge, MA: MIT Press.

Squire LR (1992) Memory and the hippocampus: a synthesis from findings with rats, monkeys, and humans. Psychol Rev 99:195-231.

Squire LR, Zola-Morgan S, Chen K (1988) Human amnesia and animal models of amnesia: performance of amnesic patients on tests designed for the monkey. Behav Neurosci 102:210-221.

Stanfield BB, Cowan WM (1982) The sprouting of septal afferents to the dentate gyrus after lesions of the entorhinal cortex in adult rats. Brain Res 232:162-170.

Staubli U, Ivy G, Lynch G (1984) Hippocampal denervation causes rapid forgetting of olfactory information in rats. Proc Natl Acad Sci USA 81:5885-5887.

Staubli U, Fraser D, Kessler M, Lynch G (1986) Studies on retrograde and anterograde amnesia of olfactory memory after denervation of the hippocampus by entorhinal cortex lesions. Behav Neural Biol 46 : $432-444$.

Tsuji S (1974) On the chemical basis of thiocholine methods for demonstration of acetylcholinesterase activities. Histochemistry 42:99_ 110 .

Tulving E, Hayman CAG, Macdonald CA (1991) Long-lasting perceptual priming and semantic learning in amnesia: a case experiment. $J$ Exp Psychol [Hum Learn] 17:595-617.

Van Hoesen GW, Hyman BT, Damasio AR (1991) Entorhinal cortex pathology in Alzheimer's disease. Hippocampus 1:1-8.

Wible CG, Shiber JR, Olton DS (1992) Hippocampus, fimbria-fornix, amygdala, and memory: object discriminations in rats. Behav Neurosci 106:751-761

Zola-Morgan S, Squire LR (1984) Prescrved learning in monkeys with medial temporal lesions: sparing of motor and cognitive skills. I Neurosci 4:1072-1085.

Zola-Morgan S, Squire LR (1985) Medial temporal lesions in monkeys impair memory on a variety of tasks sensitive to human amnesia. Behav Neurosci 99:22-34.

Zola-Morgan S, Squire LR (1986) Memory impairment in monkeys following lesions limited to the hippocampus. Behav Neurosci 100: $155-160$.

Zola-Morgan S, Squire LR (1993) Neuroanatomy of memory. Annu Rev Neurosci 16:547-563. 